\title{
Knowledge-Base Innovation - a Technology of the Future
}

Sergei Ikovenko

Invention Machine Corporation, Boston, USA

\begin{abstract}
This paper discusses the role of knowledge in product and process innovation and technology for processing semantic knowledge.
\end{abstract}

Key words: Knowledge for innovation, semantic knowledge

\section{INTRODUCTION}

Companies and research organizations today are inundated by document and data management providers offering search engines, systems, and services (at up to 5 times the product price) claiming to support front and/or back office information management; however, none addresses the unique information needs of technical knowledge workers during the early phase of product and process development - where critical decisions impacting market success are made.

The most common search engines use only keywords and contextual references to locate information. To search and capture all technical data in a problem-solution format, e.g., reduce power, improve flow, increase viscosity, would require multiple, Boolean-based search strings, a technical skill mastered by very few people in most organizations. Even if successful, these searches bring far too many non-relevant documents to the end user for review and can easily miss critical information. Most document management systems that categorize technical documents provide only limited improvement. This approach may actually impede knowledge access because categories are created according to general topics, not the technical 
problems being researched. While some vendors offer services to customize document management formats to meet specific business tasks, this approach is costly and time-intensive - and cannot keep pace with current information growth. Again, the technical end user is left out of the knowledge management equation.

Search engines may retrieve hundreds - even thousands - of documents based on keywords, but because of the keyword search format, typically retrieve only a fraction of the information needed to solve a specific problem. The more technical the content, the lower the probability of capturing relevant solutions, and the higher the probability of missing the best solution. The result - longer and more expensive development cycles.

\section{TURNING DATA AND INFORMATION INTO KNOWLEDGE FOR INNOVATION}

Semantic knowledge processing technology transforms information into knowledge by capturing problem-solution relationships in technical documents, dramatically improving problem-solving productivity and accelerating innovation for organizations, knowledge/information management teams, and technical knowledge workers, e.g., researchers, engineers, scientists, technologists, and intellectual property professionals.

The explosive growth of the Internet and intranets has escalated two major business problems to critical status: how to find and extract relevant technical knowledge from multiple, expanding information sources; and how to format and present that information so that engineers, researchers, and scientists can easily leverage it to accelerate problem solving and concept generation.

Semantic knowledge processing provides the next paradigm in information technology by "reading" technical documents using Invention Machine's knowledge capture algorithms. As it reads, it organizes the knowledge base into a functional index of problems and solutions, and presents them in a logical format tailored for technical users and enterprise information managers.

\section{SEMANTIC KNOWLEDGE PROCESSING TECHNOLOGY}

Semantic knowledge processing is the only technology that enables semantic analysis of problem-solution (cause-effect) relationships between essential words and delivers the following solutions to the stated problems: 
first, it finds all relevant documents and only relevant documents;

second, it quickly "reads" all technical content, not just keywords;

third, it automatically extracts the technical knowledge from any information source (patents, Web sites, intranet reports, e-mail messages, etc.) and creates a "problem-solution" tree for easy navigation and analysis; and finally, by analyzing the problem-solution relationships between essential words, it is able to compose a short abstract, for easy review, referencing the technical content in each document.

Relationships between words is the key differentiator. All four benefits are achieved by using a sophisticated Subject-Action-Object (SAO) engine (patent pending), in which the most unique feature is the ability to recognize cause-effect relationships between Subjects and Objects in the text.

Nothing is more important in knowledge-based operations than causeeffect relations. The reason for this is that applied "intelligence" does not actually exist without the ability to perform cause-effect analysis. For technical, scientific, and medical research, this kind of analysis is even more important because every problem has a Subject - Action - Object format as its underlying structure.

Example: Problems are often expressed as "How to" questions: How to absorb noise? "Absorb" is the Action, and "noise" is the Object. To solve this problem a proper Subject - or answer to the question "How to absorbs noise?" - is needed. The formal definition of the technical problem in this case is the following: Subject $\mathrm{X}$ - absorbs - noise; i.e., find all Subjects that can absorb noise.

By applying SAO analysis, semantic knowledge processing technology will capture only relevant cause-effect information, in this case Subjects (S) that absorb (A) noise (O), then provide a "solution" description and a hyperlink to each source document. For end users, the search engine nightmare is solved because in the majority of cases he/she does not need to run additional searches. If the engineer is looking for a new way to fight noise, he/she simply clicks on the "absorb noise" folder which includes all known solutions (all Subjects), reads the "solution" abstract, and decides which document(s) to open for a more detailed analysis (CoBrain, 1999).

Another interesting direction of knowledge-based innovation is defined by determining and using Trends of Engineering System Evolution. 
Statistically based and empirically derived trends of evolution can be used for benchmarking and development of engineering systems.

\section{TRENDS OF ENGINEERING SYSTEM EVOLUTION}

To describe laws and trends of evolution of engineering systems is not a new issue - many scientists and philosophers have tried to do it in their works. One of the most interesting approaches to develop a detailed system of trends derived from statistical analysis of the world's patent collection (Altshuller, 1985) conducted by Genrich Altshuller.

Laws and trends of engineering system evolution are analogous to Darwin's law of natural selection. There are a lot of factors that influence the development of engineering systems: accidental, financial, psychological, subjective, etc. But once created, engineering systems start competing with each other in a "Darwinian" way. The society has limited resources for the development of those systems: resources of money, time, manpower, markets, etc. Some engineering systems win this competition, some lose.

The competition is endless, and the analysis the world's patent collection showed the common mistakes of "losers" and the shared strengths of "winners". The trends of engineering system evolution reflect common rules of "survival of the fittest" and, because of the fact that they were determined from the analysis of a big statistical database, those rules are generic for various kinds of engineering systems. Being true for one class of systems they can be applied for other classes as well.

The trends have a tremendous heuristic value - engineers can use those "rules of survival" consciously for developing and improving engineering systems, hence guaranteeing their success in competition with other systems.

It was noticed that the "rules" were applied to engineering systems in a certain sequence to provide systems' incremental improvement and survivability on the market. Grouping the "rules" according to this sequence was a prototype of the trends of engineering system evolution.

The trends give us probable directions for improving an engineering system on a conceptual level - scientifically based and empirically derived recommendations that have been proved to be true for a big number of systems.

The list of "rules for survival" of engineering systems as well as trends of technology evolution are the base of one of the modules of TechOptimizer software - a comprehensive suite of knowledge-innovation tools that helps 
to state and solve engineering problems (TechOptimizer, Ref. Manual, 1999).

There are several major trends that characterize different sides and facets of the evolution of engineering systems: their state of aggregate, continuous dynamization, rhythm coordination, etc. Applying these various trends to a specific system enables engineers to conceptually predict future patents for the evolution of this system.

For example, dynamization trend describes possible ways of improving engineering systems by designing them more and more dynamized, evolving from being monolithic, rigid to devices with more degrees of freedom, more flexible and employing liquid, gaseous components and ultimately to system that are based on filed interactions (TechOptimizer, Prof. Edition, 1999).

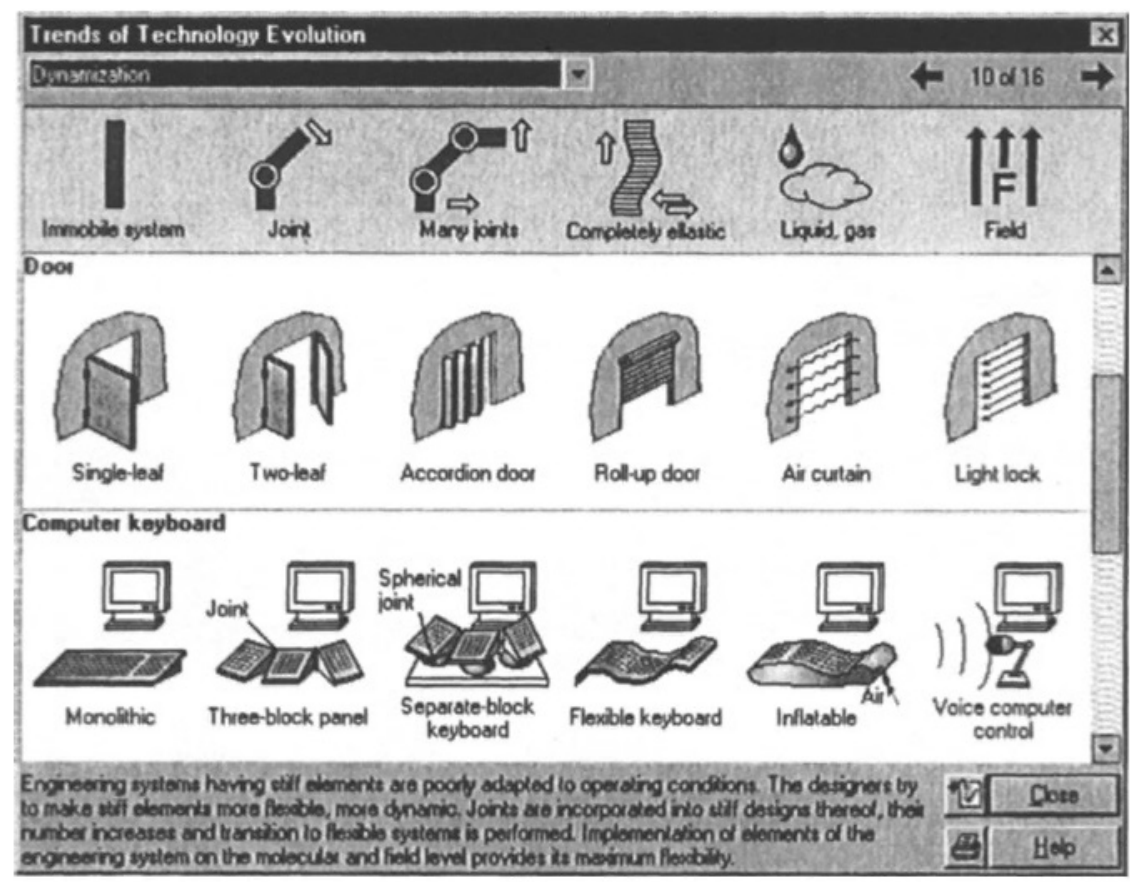

Figure 1

Applying this trend for benchmarking and analysis of mobile phones we can trace the evolution from "one rigid piece" phones to dynamized Motorola phones with joints, Alka Tel phones with liquid crystals and even predict the direction for future evolution of mobile phones.

Other trends reflect some more possible ways of evolution concerning action coordination, controllability, etc. (TechOptimizer, Prof. Ed., 1999).

For example, the so-called "mono-bi-poli" trend recommends that identical systems be combined and combinations of different (or sometimes opposite) systems follows (Fig.3). 


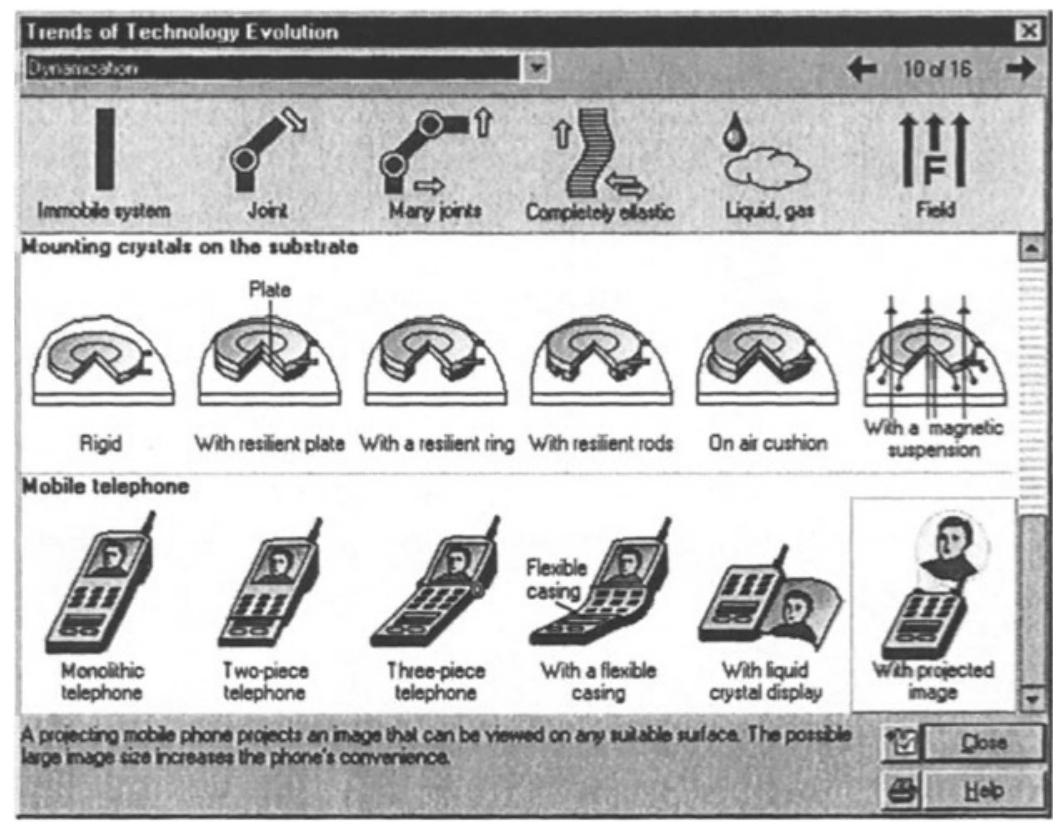

Figure 2

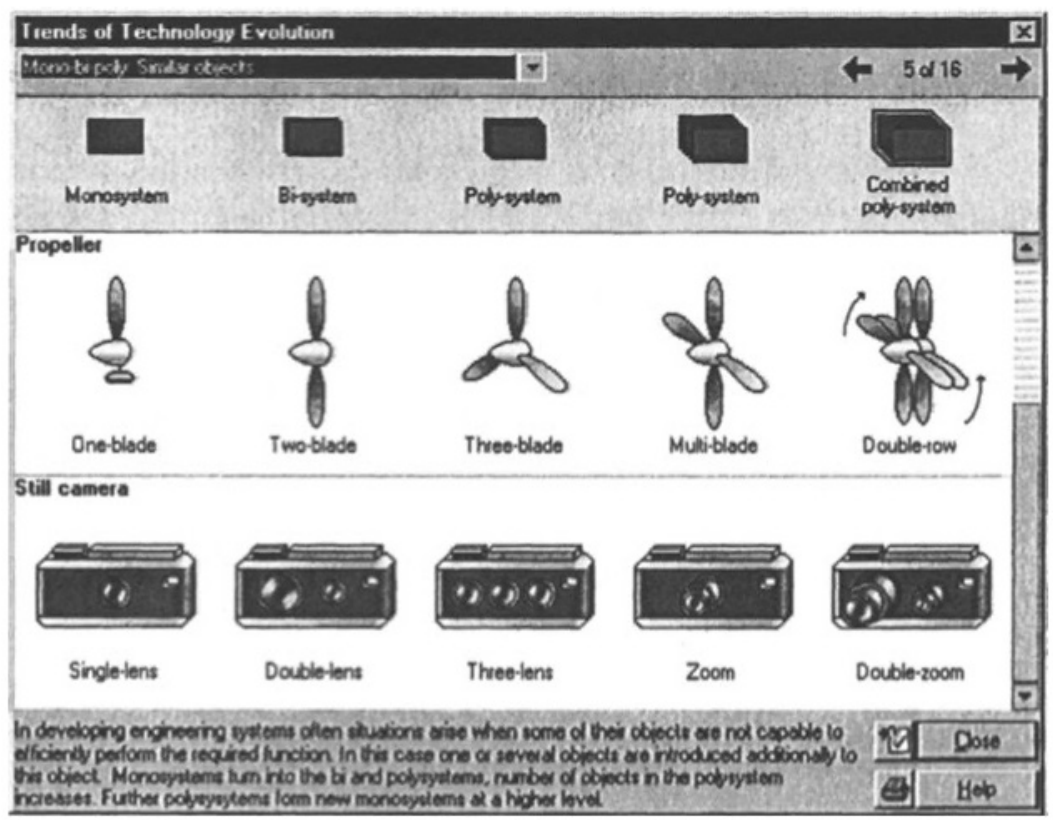

Figure 3 
If the case of cellular phones we can see the evolution from digital and analog phones to a combination of those, from one channel to multiple channels in cordless phones and in the long run - merging of these devices in one (Fig. 4).

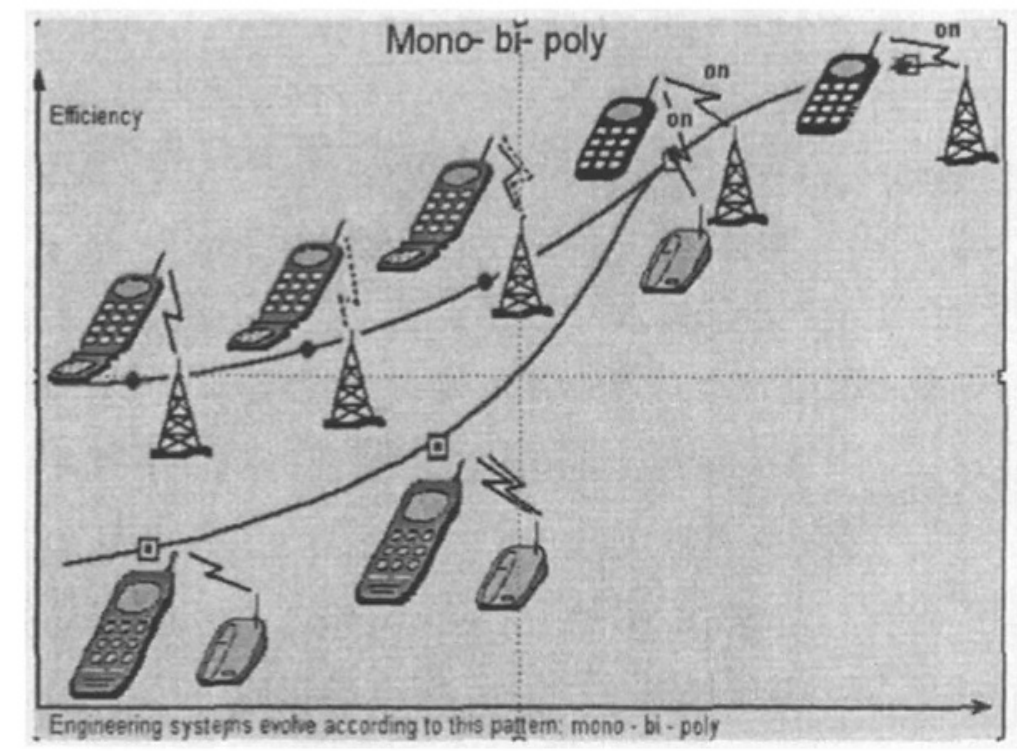

Figure 4

\section{CONCLUSIONS}

Semantic Knowledge Processing Technology. The SAO engine's ability to recognize Subject - Action - Object structures in text revolutionizes the whole process of extracting knowledge from multidisciplinary information sources. Because the engine finds problem-solution (cause-effect) relationships in texts automatically, it solves one of the most difficult challenges in knowledge management - improving the productivity of technical knowledge workers in the electronic Information Age, by capturing all relevant technical information; reducing search time by capturing only relevant information; and accelerating innovation by enabling knowledge workers to discover optimal solutions.

At the enterprise level, using the SAO engine to automate technical information processing allows corporate knowledge managers to create a single, secure knowledge base from hundreds of thousands of documents. This structured knowledge base provides access to the expertise and 
intellectual capital unique to each company and essential to its competitive future.

Building a real-time knowledge infrastructure with SAO engine applications.

An SAO engine can be used as a knowledge base creation tool for corporate Intranets, as a knowledge processor for Internet information portals, and/or as a personal knowledge assistant, to increase productivity and accelerate innovation throughout any enterprise in any industry.

Trends of Engineering System Evolution. Evolution of an engineering system in accordance with the recommendations of several trends at the same time may be compared with a movement in a multidimensional space. Knowing where the system is now and being aware of statistically proven trends of evolution, we can always outline probable directions of development and save time on trials and errors.

\section{REFERENCES}

CoBrain.com. (1999. White Paper, Invention Machine Corporation.

Genrich Altshuller. (1985). To Find an Idea, Nauka, Moscow.

Techoptimizer. (1999) Reference Manual. Invention Machine Corporation.

TechOptimizer. (1999). Professional Edition. Invention Machine Corporation. 